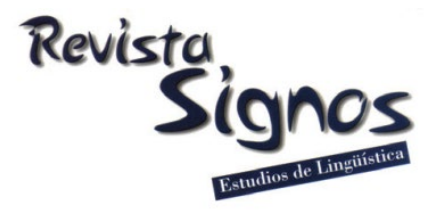

\title{
Aprendizaje simultáneo del inglés y el español en China: Una investigación basada en la Metodología-Q sobre la motivación
}

\section{Simultaneous learning of English and Spanish in China: A $Q$-method study on motivation}

\author{
Xiuchuan Lu \\ UNIVERSIDAD DE FUDAN \\ CHINA \\ luxc@fudan.edu.cn
}

\author{
Yongyan Zheng \\ UNIVERSIDAD DE FUDAN \\ CHINA \\ yongyanzheng@fudan.edu.cn
}

\author{
Wei Ren \\ UNIVERSIDAD DE BEIHANG \\ CHINA \\ weiren@buaa.edu.cn
}

Recibido: 22-VIII-2017 / Aceptado: 12-X-2018

DOI: $10.4067 /$ S0718-09342019000200381

\section{Resumen}

En la actualidad, los estudios sobre multilingüismo han captado considerablemente la atención en el campo de la lingüística, pero todavía no existen suficientes investigaciones al respecto en China. En este trabajo, mediante un experimento basado en la metodología Q, estudiaremos la disposición motivacional de un grupo de universitarios chinos que aprenden inglés y español de forma simultánea. De los resultados obtenidos, se desprende que los estudiantes chinos se han dividido en dos tipos según su actitud frente al estudio de lenguas extranjeras, que son 'solo inglés' y 'multilingüe'. Teniendo en cuenta 'el sistema motivacional del yo en L2' (L2MSS), propuesto por Dörnyei, se observa que los alumnos de 'solo inglés' están muy influenciados por 'el yo obligatorio de L2', el cual reprime su motivación para aprender español, mientras que los alumnos de 'multilingüe' buscan un camino multicultural con varias lenguas, aunque sin ideas claras ni destino concreto. Además, descubrimos que las razones que llevan a los aprendientes chinos a conocer una lengua extranjera se relacionan estrechamente con la posición social de dicha lengua en esta región.

Palabras Clave: Multilingüe, China, L2MSS, motivación, ambiente social. 


\begin{abstract}
The last fifteen years have witnessed a burgeoning interest in multilingual learning, particularly the simultaneous learning of more than one foreign language, but related research in China is limited. This paper conducted a Q-methodology investigation into a group of Chinese university students who chose to learn Spanish as their L3 in parallel with English as their L2. The results showed that Chinese students had two types of foreign-language learning motivation, namely, 'English only' and 'multilingual' motivation. Referring to the 'L2 Motivational Self System' proposed by Dörnyei, we argue that the 'English only' type was heavily influenced by their 'ought-to L2 selves', which repressed their motivation to learn Spanish, while 'multilingual' students are looking for a way towards plurilingual and multicultural competency, although most of them are yet to have clearly-defined goals. We also found that Chinese learners' choice of L3 learning is closely related to the social status of that language in this region.
\end{abstract}

Key Words: Multilingual, China, L2MSS, motivation, social context.

\title{
INTRODUCCIÓN
}

En los últimos quince años, los estudios sobre el multilingüismo han captado la atención en el campo de la lingüística, especialmente en las investigaciones acerca del aprendizaje simultáneo de dos o más lenguas extranjeras (LLEE). Algunos investigadores afirman que las personas tienen la capacidad de adquirir más de una lengua extranjera (Henry, 2014), pero también señalan que aprender una tercera (L3) es más complejo que la segunda (L2), a causa de las complejas interacciones que los diferentes sistemas lingüísticos provocan en el celebro de los aprendientes (Herdina \& Jessner, 2002; Jessner, 2006, 2008). Así, Cenoz y Genesee (1998) proponen que un estudio sobre la adquisición de L2/L3 debe tener en cuenta no solo los factores de esa lengua sino también los efectos asociados a las interacciones que produzcan otros idiomas extranjeros en el proceso de aprendizaje.

Algunos expertos que justifican dicha idea desde la perspectiva de la motivación en el aprendizaje. Sin importa cuántas lenguas extranjeras se estén aprendiendo, el proceso de su adquisición nunca ocurre de forma aislada. Además, el modo de motivar para aprender cada lengua extranjera no es homogéneo (Dörnyei, Csizér \& Németh, 2006; MacIntyre, Mackinnon \& Clément, 2009) e, incluso, en el caso de la L3, tiende a variar aún más (Mercer, 2011b). A ello se debe añadir que la disposición de los estudiantes que están experimentando el aprendizaje simultáneo de dos lenguas extranjeras se tornará aún más complicado cuando uno de los dos idiomas sea el inglés. Sabemos que como consecuencia de la globalización, hoy en día, esta lengua se ha extendido como un medio de comunicación mundial y se ha convertido en una parte fundamental de la vida cotidiana de muchas personas (Graddol, 2006). Existen investigaciones que demuestran que ella presenta un impacto negativo en el aprendizaje de otras lenguas extranjeras modernas tales como, por ejemplo, el alemán, el francés y el español (Dörnyei \& Csizér, 2002; Csizér \& Dörnyei, 2005a, 2005b; 
Csizér \& Lukács, 2010; Henry, 2010, 2011, 2014). En este sentido, por ejemplo, en Suecia, la idea de 'el inglés es suficiente' ha reducido el interés por otras lenguas extranjeras y ha debilitado su motivación en el aprendizaje (Cabau-Lampa, 2007; Henry, 2010, 2011).

La motivación y actitud de los aprendientes también se relacionan con la posición social que encuentran las lenguas extranjeras en el contexto de su aprendizaje (Henry, 2015; Huang, Hsu \& Chen, 2015). Además, los factores socioeconómicos también resultan importantes en la formación de la actitud (Coleman, Galaczi \& Astruc, 2007; Coffey, 2018). Así, el inglés tiene una gran relevancia social, teniendo en cuenta su extendido uso y su actual posición social. No obstante, dicha relevancia tiende a variar dependiendo del entorno geográfico y sociocultural. Sin embargo, hasta la fecha, la mayoría de los estudios se han realizado en contextos europeos, donde aprender varias LLEE simultáneamente es una experiencia muy común (Henry, 2015). Sin embargo, en otras partes del mundo, por ejemplo, en China, todavía se conoce muy poco sobre el motivo por el que los estudiantes quieren aprender dos o más LLEE al mismo tiempo y cómo ello influiría en sus preferencias, sus disposiciones actitudinales y motivacionales.

Teniendo en cuenta todo lo mencionado, en esta investigación se pretende estudiar el caso de un grupo de universitarios chinos que aprende español como L3 e inglés como L2. Adoptando 'el sistema motivacional del yo en L2' (L2 motivation self system) de Dörnyei (2005, 2009), que describirá la motivación en el aprendizaje de las LLEE. Investigaremos e intentaremos dar respuesta a las siguientes preguntas:

(1) Entre los aprendientes chinos que están estudiando inglés y español simultáneamente, ¿existen diferentes motivaciones en su aprendizaje? ¿Son iguales sus imágenes motivacionales basadas en 'el yo en L2' para aprender inglés y las correspondientes para aprender español?

(2) ¿Cuáles son los factores individuales, educativos y sociales que afectan a la motivación y actitud en el aprendizaje de inglés y español? ¿Cómo funciona en el aprendizaje el impacto sociolingüístico que se produce en la construcción de las auto-imágenes de los aprendientes para cada lengua específica?

\section{Estudios anteriores}

\subsection{Marco teórico: 'el sistema motivacional del yo en L2'}

Con base en las teorías psicológicas del 'yo posible' (Markus \& Nurius, 1986) y la teoría de la autodiscrepancia (Higgins, 1987), Dörnyei (2005, 2009) propone 'el sistema motivacional del yo en L2' y define a la motivación como la aspiración y la esperanza de un aprendiente para convertirse en un hablante competente de una L2 en lugar del deseo de integrarse en cierta comunidad de la L2. Ello resulta diferente 
respecto de las propuestas de Gardner y Lambert (1972) en su modelo socioeducacional.

En concreto, Dörnyei y su colega (Dörnyei, 2005; Dörnyei \& Ushioda, 2009) consideran la motivación en el aprendizaje de una L2 como un deseo del aprendiente para reducir la discrepancia entre sus posibles 'auto-guías' (self-guides) formadas por 'el yo ideal de L2' ('ideal L2 self), 'el yo obligatorio de L2' (ought-to L2 self) y 'el yo real' (real self). 'El yo ideal de L2', según Dörnyei y sus colegas (Dörnyei, 2009; Taguchi, Magid \& Papi, 2009), abarca el interés cultural, la orientación integradora y el instrumento para el mejoramiento, mientras que 'el yo obligatorio de L2' está relacionado con las necesidades externas que aún no se han internalizado, incluyendo el instrumento para la prevención, la influencia familiar y la presión causada por el ambiente. Además, existe un tercer componente importante en la motivación que es la experiencia del aprendizaje de la L2 (L2 learning experience), es decir, el entorno inmediato: la influencia del docente, el plan de estudios, el grupo de compañeros, la sensación de éxito, etc. De este modo, las auto-imágenes de los alumnos están sujetas a diversos factores de su vida y su interacción con el ambiente sociocultural. Por ello, la motivación no solo es algo individual, sino también social, la cual se integra de manera dinámica y holística (Dörnyei \& Ushioda, 2011).

\subsection{Estudios anteriores basados en 'el sistema motivacional del yo en L2'}

'El sistema motivacional del yo en L2' ya se ha aplicado en estudios donde los alumnos aprenden simultáneamente dos o más LLEE. Se han llevado a cabo una serie de investigaciones en Europa, por ejemplo, en Hungría (Csizér \& Dörnyei, 2005a, 2005b; Dörnyei, et al., 2006; Csizér \& Lukács, 2010), Suecia (Henry, 2010, 2011, 2014, 2015) y Austria (Mercer, 2011a, 2011b), las cuales reflejan que el inglés puede funcionar como una referencia normativa teniendo una influencia negativa en otras LLEE, debido a la excesiva dedicación y recursos que emplean los estudiantes en ella. Henry (2014) apunta que las auto-imágenes de las LLEE están relacionadas con la posición social de la lengua en un contexto específico. Así, en una sociedad donde la L2 (inglés) tiene una alta posición social y amplia presencia, esta puede tener impactos negativos en la motivación de una L3 menos corriente y menos prestigiosa. Esta propuesta la afirma Busse (2017) a través de una serie de encuestas realizadas en cuatro países europeos.

Sin embargo, fuera del contexto europeo, encontramos escasos estudios que hayan estudiado la motivación de los estudiantes dedicados al aprendizaje simultáneo de las LLEE bajo ese sistema teórico de Dörnyei. Al respecto, Yashima (2002, 2009) señala que el yo ideal de L2 (inglés) de los aprendientes japoneses se relaciona con la posición importante que ocupa en la comunidad internacional. Ellos muestran un gran interés por asuntos internacionales con ganas de ir al extranjero a estudiar y trabajar. Lamb 
(2004, 2009), con sus estudios en Indonesia, concluye que los estudiantes asiáticos aspiran a integrarse en una comunidad internacional imaginaria mediante la construcción de una identidad ciudadana global del habla inglesa. Por su parte, Siridetkoon y Dewaele (2017) indica que en Tailandia el inglés amenaza la motivación de los alumnos para estudiar otros idiomas extranjeros, pero que -al mismo tiempolos alienta a estudiar esos idiomas. Los aprendientes de lenguas extranjeras múltiples desarrollan su único sistema motivacional en que las interrelaciones de esas motivaciones múltiples son dinámicas y complejas y, además, tienen su motivación en la necesidad inmediata y el futuro uso. Huang et al. (2015), con sus cuestionarios cuantitativos, demuestran que los alumnos taiwaneses de LLEE están influenciados por una identidad cultural y socialmente tradicional. Así, por ejemplo, los que aprenden japonés y alemán siempre tienen una fuerte motivación instrumental, mientras que el interés personal por la cultura y la propia voluntad por aprender juegan un papel más decisivo en el aprendizaje del francés y el coreano.

Existen otros que han presentado el yo de L2 y la motivación de los aprendientes de L2 en el contexto de Asia (véanse más datos en Apple, Da Silva \& Fellner, 2013, 2016). Sin embargo, la mayoría de ellos tienen el enfoque principal centrado en el aprendizaje del inglés. Todavía faltan investigaciones en las que se observe cómo son las auto-imágenes específicas de diferentes LLEE y su relación con la posición de dichas lenguas en el mismo contexto sociocultural, sobre todo, en China.

\subsection{El aprendizaje del inglés en China}

Hoy en día, al ser una lengua global, el inglés se usa cada día más en todo el mundo (Crystal, 2003; Graddol, 2006), convirtiéndose también en un idioma extranjero dominante en China. Considerando el poder y la influencia de este idioma y su papel indispensable en el proceso acelerador de la globalización, las autoridades chinas están muy motivadas para llegar al nivel internacional y están promoviendo esta lengua en la educación secundaria y superior con el fin de ampliar y profundizar la participación de este país en las actividades políticas, económicas y culturales de la comunidad internacional (Lam, 2002; Pan, 2011; Bolton \& Graddol, 2012). Por su parte, los estudiantes también ven dicha lengua como la clave para alcanzar oportunidades socioeconómicas y educativas (Hu, 2003; Gao, 2008; Pan \& Block, 2011). Bajo este ambiente socioeconómico, el inglés se ha posicionado como el idioma extranjero más importante e, incluso, dominante en el sistema educativo chino. Según la reciente encuesta de Wei y Su (2012), hay 416 millones de estudiantes que aprenden lenguas extranjeras. Entre ellos el 93,8\% estudian inglés, el 7,1\%, ruso, el 2,5\%, japonés y solo el $0,3 \%$ están aprendiendo otras lenguas extranjeras. Tal y como propone Dörnyei y Al-Hoorie (2017), el aprendizaje de inglés está principalmente institucionalizado mientras que el de otras lenguas extranjeras puede estar desequilibrado en diferentes contextos. 
A pesar de su destacado papel en el país oriental, el inglés realiza, la mayor parte de las veces, funciones instrumentales en el campo educativo (Feng, 2009) con 'fines intranacionales' (intra-national purposes) (Bolton \& Graddol, 2012). No obstante, la función comunicativa de este idioma ha pasado a un segundo plano, debido a la presión que ejercen los exámenes sobre los estudiantes que deben realizar estas pruebas para obtener sus pertinentes títulos, ya que existe una idea reinante: exámenes primero (Pan \& Block, 2011).

La descripción anterior pone de manifiesto varias características del contexto chino en comparación con el europeo. Por un lado, a diferencia del entorno europeo, donde aprender varias lenguas extranjeras es algo habitual (Henry, 2015), el inglés tiende a ser la única lengua extranjera que los chinos estudian en la escuela; por otro lado, las orientaciones políticas de las lenguas extranjeras son muy distintas. Asimismo, las políticas educativas de la Unión Europea consideran el desarrollo del multilingüismo como un objetivo principal (Cenoz \& Jessner, 2000; Henry, 2010), tal y como se refleja en la Estrategia de Lisboa, mientras que en China tienden a mantener la enseñanza del inglés como un contenido esencial, casi ignorando por completo otras lenguas extranjeras. Teniendo esto presente, estudiar la motivación y actitud de los aprendientes chinos sobre las diferentes lenguas extranjeras podrá arrojar información útil y valiosa en cuanto a la naturaleza interactiva y situacional que alienta al estudio de la L2 y la L3.

\subsection{El español y su aprendizaje en China}

El español es el segundo idioma con mayor número de hablantes en el mundo (después del chino). Según el Instituto Cervantes (2016), es la segunda lengua en el cómputo global de hablantes y ocupa también el segundo puesto en la clasificación de idiomas más estudiados como segunda lengua, junto con el francés y el chino mandarín.

Con respecto a la motivación en el aprendizaje del español, la mayoría de los estudios que se han llevado a cabo en Europa o Estados Unidos se han centrado en describir los diferentes tipos (basados en Gardner \& Lambert, 1972), las actitudes de los alumnos, así como la correlación entre dichas variables. Mediante cuestionarios, descubrieron que los estudiantes, en dichos contextos, cuentan con mayor motivación integradora que instrumental (Espí \& Azurmendi, 1996; Minera, 2009; Fonseca \& García, 2010). Asimismo, existen tratados que afirman que la motivación para aprender español puede y suele variar con el paso del tiempo y con el cambio de contexto al tomar este idioma como una seña de identidad, que puede facilitar la inserción de un miembro en la comunidad hegemónica, permitiendo la cohesión grupal en contextos multiculturales (Segalowitz \& Freed, 2004; Carrai, 2009). Las investigaciones realizadas en Europa y Estados Unidos llegan a la conclusión de que muchos de los aprendientes de español en esas zonas tienen como incentivo la 
'integratividad' (integrativeness). Pero nos interesaría saber cómo es la situación del aprendizaje del español en China, al ser este un país geográfica y culturalmente tan lejano a Europa y Estados Unidos.

A medida que se desarrolla la relación político-económica entre China y el mundo hispánico, sobre todo con América Latina, va aumentando la demanda de personas que saben dicha lengua concienciándose "progresivamente del significado y el valor del idioma español como activo y recurso en el mercado laboral" (González Puy, 2006: 133). En la actualidad, hay más de cincuenta mil estudiantes de esta lengua, incluyendo los matriculados en la especialidad universitaria y los que la aprenden como una afición en su tiempo libre (Zheng, 2015). Sin embargo, en comparación con la situación en Europa y en EEUU, no hay muchos estudios que investiguen las motivaciones de los chinos para aprender español ni que revelen la interacción entre aquellos y el particular ambiente socio-político de esta región, sin hablar de los que están aprendiendo dos lenguas extranjeras de forma simultánea.

\section{El estudio}

\subsection{Contexto y participantes}

El presente estudio se ha realizado en la Facultad de Lenguas Extranjeras y Literatura de una de las universidades más prestigiosas de China. Los participantes son 35 universitarios de especialidad inglesa que aprenden español como L3. Estos alumnos asisten a un curso intensivo de español durante un año (en su segundo curso académico universitario). En total, reciben unas 250 horas lectivas de español y también dedican tiempo fuera de clase para repasar y progresar en este idioma. En cuanto al estudio del inglés, le dan la misma importancia porque la filología inglesa es su especialidad universitaria. Por eso, consideramos el caso como un aprendizaje simultáneo del inglés y el español. Hay que señalar que, según Ortega y Byrnes (2008), estos alumnos tienen una experiencia de más de siete años aprendiendo la lengua anglosajona y han adquirido un nivel equivalente al B2-C1. He aquí los datos demográficos de los participantes:

Tabla 1. Datos demográficos de los participantes.

\begin{tabular}{|l|l|}
\hline & Participantes \\
\hline Descripción & $\begin{array}{l}\text { Estudiantes de segundo año académico del departamento de inglés } \\
\text { que eligen voluntariamente español como L3 }\end{array}$ \\
\hline Género & Hombres $=6$ Mujeres $=29$ \\
\hline Edad & $18-20$ \\
\hline L1 & Chino \\
\hline L2 & Inglés \\
\hline L3 & Español (dos meses de aprendizaje desde el inicio) \\
\hline $\begin{array}{l}\text { Conocimiento de inglés y el } \\
\text { nivel esperado }\end{array}$ & $\begin{array}{l}8-10 \text { años de aprendizaje; } \\
\text { Un nivel de español equivalente al B2-C1 de acuerdo con el MCER }\end{array}$ \\
\hline
\end{tabular}




\subsection{Metodología}

'La metodología Q', utilizada en la presente investigación, es un sistema propuesto inicialmente por Stephenson en el año 1935. Se trata de una innovadora técnica en el campo de investigaciones sociocientíficas, sobre todo en lingüística. Esta permite revelar las subjetividades desde el punto de vista de los propios aprendientes. Pese a ser una metodología cualitativa, emplea una base estadística sólida (análisis factorial) con el fin de analizar los efectos de la información obtenida cualitativamente (Brown, 1995). Su elemento más característico es la medición objetiva de la subjetividad (Watts, 2015). En palabras de Valencia Vallejo (2003: 146), 'la metodología Q' "revela ordenamientos por rango de la opinión de las personas". Además, se trata de someter a un número reducido de personas un gran número de propuestas y no lo contrario (Prou, Le Nedic \& Amslem, 2009). Por eso, esta metodología es muy apropiada para el presente estudio, que se realiza con una pequeña muestra de participantes y con el propósito de medir y clasificar la subjetividad de ellos.

'La metodología Q' se ha aplicado con éxito a los estudios de ciencia política y de educación (Yang \& Montgomery, 2013) y, recientemente, se ha introducido como un método innovador en las investigaciones de motivación en L2 (Irie \& Ryan, 2015; MacIntyre, MacKay, Ross \& Abel, 2017).

Necesitamos llevar a cabo cuatro pasos para realizar un estudio con la metodología Q: (1) desarrollar los enunciados (statements) (normalmente varía de 40 a 80 enunciados) para construir una miniatura del conjunto de saberes compartidos; (2) recopilar las opiniones de los participantes, lo que llamamos 'clasificación Q'; (3) hacer un análisis factorial de dicha 'clasificación Q'; (4) interpretar los factores emergentes en relación con los enunciados. Los factores representan las distintivas opiniones compartidas dentro del grupo de participantes; eso quiere decir que los individuos se agrupan según sus puntos de vista compartidos sobre cierto tema. Un estudio de metodología Q, en general, tiene entre 20 a 40 participantes según los diferentes casos (Watts, 2015).

Además, para enriquecer el resultado obtenido con la metodología Q, también realizamos entrevistas con los participantes después del experimento, tomándolas como un método suplementario para comprender y explicar mejor su motivación. Esto es porque, después de obtener la medición y clasificación objetiva de la subjetividad de los participantes, necesitamos comprender con profundidad lo que piensan los participantes con el fin de explicar sus opciones en el experimento. Para llevar a cabo las entrevistas, primero, planificamos todas las preguntas que queremos formular y preparamos un guion (véase el guion en Anexo II). Las preguntas que se realizan son abiertas y relacionadas estrechamente con la actitud y motivación de los alumnos hacia el aprendizaje de inglés y español, el participante puede expresar sus opiniones o hacer comentarios. Invitamos a los 35 participantes, uno tras otro, a un 
despacho cerrado y tranquilo, en el que se sientan cómodos, les hacemos las preguntas según el guion cara a cara con una grabadora para realizar la grabación de la conversación y facilitar posteriormente su transcripción. Todo el proceso lo realizamos en el idioma chino para que los participantes entiendan y se expresan con mayor facilidad. La entrevista dura unos 20 minutos por cada participante.

\subsection{Desarrollo del experimento}

El presente estudio se realiza siguiendo los pasos principales de la metodología Q (Watss \& Stenner, 2012). Primero creamos una lista de 60 enunciados (véase Anexo I). Estos enunciados se elaboran de acuerdo con los estudios anteriores de motivación en L2 de Csizér y Dörnyei (2005a, 2005b), y Dörnyei et al. (2006). Todos ellos recopilan motivaciones y actitudes muy diferentes y representativas frente al aprendizaje del inglés y el español, no solo recogiendo posturas ordinarias sobre las lenguas y su cultura, sino también incluyendo los valores lingüísticos, culturales y sociales. Cada enunciado se ha impreso en una tarjeta de papel ( $90 \mathrm{~mm}$ x $54 \mathrm{~mm})$ en chino.

Segundo, pedimos a los participantes hacer la 'clasificación Q' durante una clase de español (45 minutos). A cada participante se le entrega un juego de tarjetas (cada juego contiene 60 tarjetas de los 60 enunciados) y se le pide que clasifique los 60 enunciados puestos en la tarjeta en función del grado de acuerdo o desacuerdo (de menos seis a más seis-trece posibilidades--), y que rellene el número de cada enunciado en un formulario de distribución forzada (Gráfico 1). Los 35 participantes en total generan 35 'clasificaciones Q'.

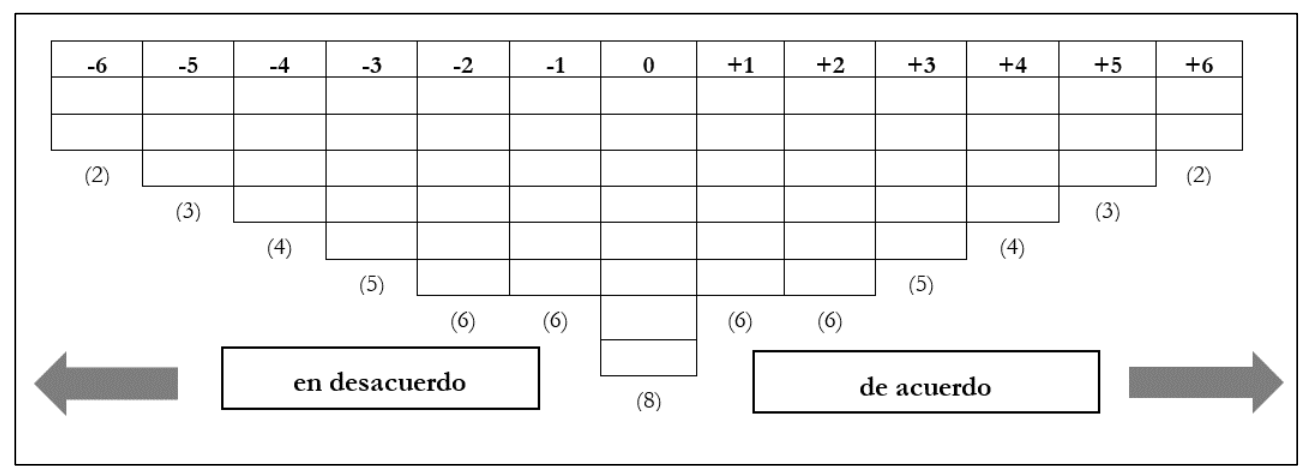

Gráfico 1. Cuadro de aplicativo de metodología Q.

Tercero, utilizamos el PQMethod (www.rz.unibwmunchen.de/ p41bsmk/qmethod), un software gratuito, para realizar el análisis factorial requerido por la metodología Q. Ingresamos las 35 'clasificaciones Q' en este software y se obtiene una matriz de correlación entre esas clasificaciones a través de la extracción del centroide. Luego, la matriz de correlación se entrega al análisis del componente principal con una rotación varimax (normalización de Kaiser). Después 
del ensayo de las soluciones de uno, dos y tres factores, encontramos que la de dos factores llega a ser más apropiada basada en la varianza explicada del 48\%, lo cual cumple con los requisitos de la metodología Q (Watts \& Stenner, 2012). Las cargas se consideran estadísticamente significativas con $\alpha=0.01, \mathrm{r}>.43$ (Watts \& Stenner, 2012). Es decir, la solución final identifica dos grupos de factores significativos, con diecinueve clasificaciones en Factor 1, que representa el $42 \%$ de la varianza y nueve en Factor 2, que representa el 6\% de la varianza. Existen, además, seis clasificaciones cargadas en ambos factores y una sin posibilidad de ser cargada, casos que en el presente estudio no van a ser investigados.

\section{Resultados}

Según las clasificaciones individuales y la configuración general de los enunciados capturados en la matriz, los dos grupos recibieron las siguientes etiquetas descriptivas: Factor 1 (solo inglés) y Factor 2 (multilingüe). Los dos factores representan diferentes tipos de actitud frente a la evaluación de los enunciados. La distribución de los participantes en dichos grupos se representa en la siguiente Tabla:

Tabla 2. Integración de los factores.

\begin{tabular}{|l|l|}
\hline \multicolumn{1}{|c|}{ Grupos } & \multicolumn{1}{|c|}{ Clasificaciones Q que integran el grupo } \\
\hline Factor 1 (solo inglés) & Q1, Q2, Q3, Q4, Q6, Q11, Q14, Q15, Q16, Q18, \\
& Q19, Q20, Q24, Q25, Q28, Q29, Q30, Q33, Q35 \\
\hline Factor 2 (multilingüe) & Q5, Q7, Q10, Q21, Q26, Q27, Q31, Q32, Q34 \\
\hline Grupo con cargas dobles & Q8, Q9, Q12, Q17, Q22, Q23 \\
\hline Imposible de ser cargado & Q13 \\
\hline
\end{tabular}

En este momento, se interpretan los resultados obtenidos comparando los enunciados extremos que definen a cada uno de los factores y, así, se diferencian.

\subsection{Factor 1: 'Solo inglés'}

Según el resultado que obtenemos, los enunciados marcados con +6 , +5 , -6 y -5 para el grupo del Factor 1 (solo inglés) son los siguientes: 
Tabla 3. Enunciados extremos seleccionados por el Factor 1.

\begin{tabular}{|c|c|c|c|}
\hline & Enunciados distintivos en Factor 1 & Rango & Valor normal $^{1}$ \\
\hline \multirow{5}{*}{ De acuerdo } & 5. Continuaré aprendiendo inglés en el futuro. & +6 & 1.943 \\
\hline & $\begin{array}{l}\text { 16. Tengo que aprender bien el inglés, si no, afectará } \\
\text { mis calificaciones. }\end{array}$ & +6 & 1.677 \\
\hline & $\begin{array}{l}\text { 48. Me gustan los productos culturales de los países } \\
\text { anglo-americanos, tales como la música, las series o las } \\
\text { películas. }\end{array}$ & +5 & 1.580 \\
\hline & $\begin{array}{l}\text { 24. Me gustan mucho las clases de español que estoy } \\
\text { cursando. }\end{array}$ & +5 & 1.323 \\
\hline & $\begin{array}{l}\text { 55. Espero ir algún día a los países angloamericanos } \\
\text { para hacer intercambios o para vivir. }\end{array}$ & +5 & 1.321 \\
\hline \multirow{5}{*}{$\begin{array}{c}\text { En } \\
\text { desacuerdo }\end{array}$} & $\begin{array}{l}\text { 33. Al encontrarme con dificultades en el estudio de } \\
\text { inglés, siempre pienso: "No pasa nada, no sería } \\
\text { importante si no pudiera aprender bien las LLEE." }\end{array}$ & -6 & -2.241 \\
\hline & $\begin{array}{l}\text { 19. Tengo que aprender bien español, si no, no podré } \\
\text { encontrar un buen trabajo en el futuro. }\end{array}$ & -6 & -1.876 \\
\hline & $\begin{array}{l}\text { 2. Prefiero dedicar más tiempo y esfuerzo al estudio del } \\
\text { español. }\end{array}$ & -5 & -1.678 \\
\hline & $\begin{array}{l}\text { 8. Siempre asigno una gran parte de mi tiempo, } \\
\text { primero al estudio del español, y el resto al inglés. }\end{array}$ & -5 & -1.573 \\
\hline & $\begin{array}{l}\text { 53. Espero que algún día pueda ser personas a estilo de } \\
\text { los hispanohablantes. }\end{array}$ & -5 & -1.476 \\
\hline
\end{tabular}

Los estudiantes de este grupo subrayan la importancia del aprendizaje del inglés, diciendo que continuarán aprendiendo esta lengua en el futuro (enunciado 5) y también muestran su deseo de ir a países angloamericanos para tener la experiencia vital allí (enunciado 55). Además, los miembros del Factor 1 toman el estudio de inglés como una tarea muy importante porque se relaciona estrechamente con sus calificaciones universitarias (enunciado 16). La mayoría pretende conseguir buenas notas. Tal y como dicen los participantes:

Q6: "Dedico más tiempo al estudio del inglés, si no, voy a fallar en los exámenes."

Q16: "Estudio tanto inglés porque quiero tener buenas notas, ya que estas me proporcionarán muchas ventajas en todos los aspectos."

Aparte, los factores culturales, sin duda alguna, juegan un papel determinante a la hora de estimular a los estudiantes. Los alumnos de este grupo declaran que solo les gustan aspectos culturales de Estados Unidos y Reino Unido, afirmación que realizan sin tener conocimientos de otros países angloparlantes:

Q3: “A mí me gustan los cantantes estadounidenses; están muy de moda. Pero no conozco mucho la música de otros países de habla inglesa, en realidad, casi nada."

Q16: "Veo muchas películas y series estadounidenses; Ahora en China se pueden encontrar un motón de productos culturales de Estados Unidos." 
Si nos fijamos en el enunciado 24 (véanse Tablas 3 y 4), los dos grupos lo marcan con puntuaciones altas (Factor 1: +5 y factor 2: +6 ). Cabe preguntarse si a los estudiantes del Factor 1 de verdad les interesa el estudio del español y, si es así, ¿por qué les denominamos como el grupo de ‘solo inglés'? Al ver los enunciados marcados con -6 y -5, nos damos cuenta de que, en efecto, no le dan mucha importancia al estudio del español. Como ya tienen un nivel de inglés bastante alto, no piensan que este idioma tenga algún vínculo directo con su futura profesión (enunciado 19). Así, por ejemplo, el participante Q33 dice:

"Todavía quedan dos años para graduarme. Para entonces, ¿quién sabe qué nivel de español puedo tener? No creo que me vaya a ayudar mucho."

Además, siempre prefieren dedicar más tiempo y esfuerzo a la lengua anglosajona (enunciado 2, 8), diciendo que solo cuando tengan tiempo libre, repasarán lo que hayan aprendido de español.

Aquí se hacen evidentes las diferencias de los estudiantes del Factor 1 en cuanto a su actitud frente al inglés y al español. Los alumnos se exigen mucho a ellos mismo en el estudio del inglés, sin abandonar cuando se encuentran con dificultades (enunciado 33), demostrando una fuerte voluntad y una gran persistencia (enunciado 5). Sin embargo, el estudio del español lo toman como una afición para el tiempo libre y no como una tarea obligatoria.

MacIntyre et al. (2009) hacen hincapié en las diferencias interculturales y su impacto en las motivaciones de los estudiantes. Teniendo esto en cuenta, Huang et al. (2015) señalan que en muchos países de Asia Oriental el confucianismo influye profundamente en el autoconcepto y en el comportamiento de la gente. En esta corriente filosófica tradicional son importantes los altos logros académicos y el deber o la obligación de los individuos en la sociedad. El conocimiento de LLEE, en especial del inglés, siempre ha sido considerado como una demostración de dicho alto logro académico. Por esta razón, cuando los estudiantes pueden conseguir éxitos en el estudio del inglés, experimentan una autosatisfacción y un reconocimiento popular y social, que repercute en su búsqueda de trabajo en el futuro.

Los miembros del Factor 1, aunque están aprendiendo español, siguen valorando el inglés como la LE más importante, por las ventajas que supone socialmente en China y porque supone un medio insustituible para su autosatisfacción y su futura profesión. Por ello, este grupo tiene una motivación muy fuerte para aprender inglés. Además, siempre relacionan su motivación con el inglés estándar del Reino Unido y Estados Unidos.

Los estudiantes de este grupo, en efecto, no tienen formado el concepto del 'inglés global', ni tampoco tiene una idea muy clara sobre la posición social de este idioma en 
el futuro. Asimismo, se caracterizan por seguir obedeciendo la tradición de 'una nación, una lengua', sin ver cada idioma como uno de los recursos multilingües. Es decir, el inglés en vez de ser tomado como un instrumento de comunicación global, continúa siendo considerado como un elemento académico individual. En cuanto al español, los estudiantes del Factor 1 no demuestran un verdadero interés por esta lengua, sino que la perciben como una vía para ayudarles a alcanzar su autosatisfacción.

Por otra parte, el hecho de que a este grupo de estudiantes le encante sus clases de español revela que su actitud frente al aprendizaje de LLEE también tiene que ver con su ambiente de estudio (profesores, libros de texto, actitud de los compañeros, entorno de estudio, etc.). Tal y como proponen Dörnyei y Ushioda (2011), la influencia del ambiente es un proceso 'de abajo arriba'. Sin embargo, es muy difícil que este se convierta en un elemento crucial para reforzar o cambiar la motivación de los alumnos.

\subsection{Factor 2: 'Multilingüe'}

Los enunciados marcados con $+6,+5,-6$ y -5 , que reflejan las actitudes extremas y más típicas de los estudiantes de Factor 2, son los siguientes:

Tabla 4. Enunciados extremos seleccionados por el Factor 2.

\begin{tabular}{|c|c|c|c|}
\hline & Enunciados distintivos en Factor 2 & Rango & Valor normal \\
\hline \multirow{5}{*}{ De acuerdo } & $\begin{array}{l}\text { 24. Me gustan mucho las clases de español que estoy } \\
\text { cursando. }\end{array}$ & +6 & 1.943 \\
\hline & 43. El español me parece muy interesante. & +6 & 1.765 \\
\hline & $\begin{array}{l}\text { 56. Espero que algún día pueda ir a los países } \\
\text { hispanohablantes para hacer intercambios o para vivir. }\end{array}$ & +5 & 1.637 \\
\hline & $\begin{array}{l}\text { 10. Puedo imaginar que en el futuro podré usar el inglés } \\
\text { sin ningún problema en algún trabajo o en la vida } \\
\text { cotidiana. }\end{array}$ & +5 & 1.533 \\
\hline & $\begin{array}{l}\text { 54. Creo que para adquirir una lengua también hace falta } \\
\text { conocer su cultura }\end{array}$ & +5 & 1.393 \\
\hline \multirow{5}{*}{$\begin{array}{c}\text { En } \\
\text { desacuerdo }\end{array}$} & $\begin{array}{l}\text { 44. He decidido aprender español porque es una lengua } \\
\text { relativamente fácil. }\end{array}$ & -6 & -2.001 \\
\hline & $\begin{array}{l}\text { 38. Como el inglés es la lengua franca o vehicular en } \\
\text { todo el mundo, sería suficiente aprender solo esta lengua. }\end{array}$ & -6 & -1.893 \\
\hline & $\begin{array}{l}\text { 32. Al encontrarme con dificultades en el estudio del } \\
\text { español, siempre pienso: "No pasa nada; por lo menos } \\
\text { hablo bien el inglés." }\end{array}$ & -5 & -1.857 \\
\hline & $\begin{array}{l}\text { 33. Al encontrarme con dificultades en el estudio del } \\
\text { inglés, siempre pienso: "No pasa nada, no sería } \\
\text { importante si no pudiera aprender bien las LLEE." }\end{array}$ & -5 & -1.847 \\
\hline & $\begin{array}{l}\text { 20. Como hablo bien el inglés, no es importante si no } \\
\text { puedo aprender bien el español. }\end{array}$ & -5 & -1.790 \\
\hline
\end{tabular}

Para los estudiantes de este factor, el español, es de gran interés. Les parece un idioma con encanto (enunciado 43) y su estudio representa un proceso divertido con 
el que disfrutan mucho (enunciado 24). No solo expresan una actitud positiva, sino que también demuestran ganas de conocer la sociedad hispánica (enunciado 56). Así, por ejemplo, manifiestan el deseo de ir a países hispanohablantes para acercarse a su cultura. Algunos de los miembros del Factor 2 señalan en la entrevista sobre el español y su estudio lo siguiente:

Q10: "El español es muy interesante y la clase también. Puedo sentirme satisfecho después de haber empezado aprender esta lengua."

Q34: "Me gusta el español. De hecho, no conocía mucho este idioma antes. El curso de español es una oportunidad que nos ofrece la facultad."

En la entrevista, también se perciben sus actitudes positivas frente a la sociedad hispánica y su deseo de experimentar dicha cultura. Por ejemplo, algunos dicen lo siguiente:

Q7: “La cultura de hispánicas me parece muy atractiva. Las personas de España o de América Latina son entusiastas. Me alegra poder tener una amistad con ellos.”

Q32: "La cultura hispánica es muy misteriosa. Me gustaría poder conocerla más, pero en China no hay tantos productos culturales hispanos como ingleses."

Además de expresar su gusto e interés por el estudio de este idioma, los miembros del Factor 2, al igual que los del Factor 1, también señalan la importancia del inglés en su vida universitaria y expresan su deseo de usar el inglés en el futuro (enunciado 10).

Con respecto a los enunciados con los que los estudiantes del Factor 2 están más en desacuerdo, destaca el 38, que justifica su actitud multilingüe. Así, Q21 dice:

"El mundo no es el mundo del inglés, sino el mundo de muchos países y naciones. No debería existir solo una lengua y una cultura."

Podemos ver que los estudiantes del Factor 2 no rechazan la importancia del inglés, pero hacen hincapié en la coexistencia de las diferentes culturas y lenguas. Asimismo, el resultado revela que no quieren prestar solo atención y esfuerzo al estudio del inglés, sino que prefieren adquirir una L3 en cuyo proceso ansían obtener buenos resultados y éxitos (enunciado 20). También consideran que el inglés y el español poseen la misma importancia y lo manifiestan empleando su energía en el estudio de ambas (enunciado 32, 33).

En conclusión, los estudiantes de este factor se identifican como un grupo típico de actitud multilingüe. Esto quiere decir que ellos reconocen que el mundo actual no es monista, refiriéndose tanto a la lengua como a la cultura. Para ellos, es necesario conocer y aprender más de dos lenguas extranjeras para integrarse en la sociedad global y multicultural, y para la autorrealización en aspectos como el conocimiento, la profesión y la vida cotidiana. Sin embargo, según el contenido de nuestra entrevista no 
tienen una idea muy clara o un concepto concreto sobre el español, la L3 que están aprendiendo. Solo la estudian porque les ofrecen esta oportunidad, pero después de empezar a aprenderla, comprenden su relevancia en el mundo y les interesa de forma creciente su cultura por la influencia del contexto de aprendizaje, lo cual también refuerza su actitud multilingüe.

\subsection{Ideas compartidas y diferentes entre el Factor 1 y 2}

Según el resultado de nuestro experimento, los estudiantes de los dos factores, hasta cierto punto, comparten algunas ideas y presentan actitudes similares. Por ejemplo: a ambos los dos les gustan sus clases de español; admiten que en el mundo se necesitan diferentes lenguas; no quieren convertirse en personas a estilo de los hispanohablantes; no se rinden fácilmente cuando se encuentren con dificultades en el estudio del inglés; tampoco cuentan con una motivación muy instrumental en el aprendizaje de este idioma, porque no tienen ganas de participar en exámenes oficiales de español o de obtener algún certificado de la lengua. Además, los dos grupos de estudiantes comparten ciertas opiniones: les gustan sus clases de español; en el mundo se necesitan diferentes lenguas; no quieren convertirse en personas como los hispanohablantes, y no se rinden fácilmente cuando se encuentren con dificultades en el estudio del inglés. Estas creencias compartidas por los dos grupos señalan que ambos admiten la necesidad de que existan diferentes lenguas en el mundo, aunque el grupo del Factor 1 siempre tome el inglés con preeminencia.

Lo que nos llama mucho la atención es que el factor 'solo inglés' y el 'multilingüe' tienen en cuenta la existencia de las diferentes lenguas en el mundo y son conscientes de su necesidad real. Además, expresan la envidia que tienen hacia las personas que puedan hablar varias lenguas extranjeras, aunque los estudiantes del Factor 1 insisten en que en China con solo aprender bien el inglés ya se puede alcanzar el éxito académico o cierta posición profesional o social, sin mostrar mucho interés ni dedicar demasiado esfuerzo al estudio de otras LLEE, en nuestro caso, el español. En cambio, los estudiantes de 'multilingüe', a pesar de que estén en el mismo contexto social que los estudiantes de 'solo inglés', presentan un gran interés por el aprendizaje de otras LLEE, contando con una disposición motivacional y actitudinal muy diferente, e incluso proponen que el español les podrá ampliar sus horizontes presentándoles un mundo culturalmente interesante. No obstante, ninguno de los dos grupos quiere integrarse tanto que su personalidad mute a una hispanohablante, sino viendo la cultura hispánica desde un segundo plano. Es decir, que aprenden español no por las ganas de integración en la sociedad hispanohablante, lo cual es totalmente diferente de lo que indican la mayoría de los anteriores estudios, que han investigado la motivación de los estudiantes de español en Europa y Estados Unidos. 


\section{Discusión}

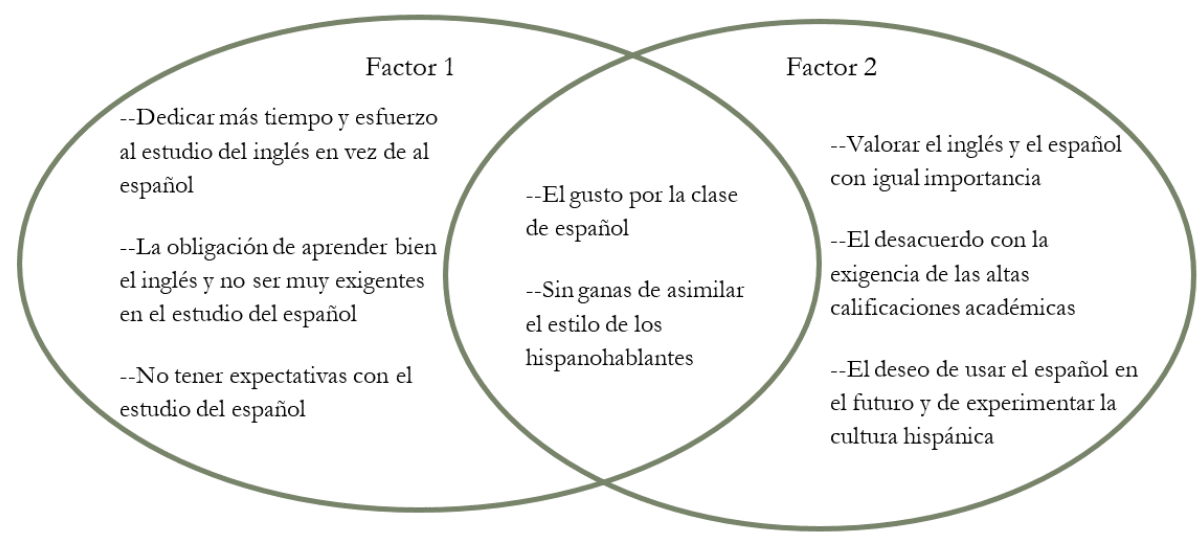

Gráfico 2. Resumen de las ideas y actitudes de los dos factores.

A partir de los mencionados análisis, nos damos cuenta de que existen grandes diferencias entre los estudiantes de los dos factores, aunque también comparten características similares, como se concluye en el Gráfico 2 de arriba. Según él, los dos grupos tienen una distribución muy distinta en lo relativo al tiempo dedicado a las dos lenguas. Así, el conjunto del Factor 1, bajo la influencia de la tradición confuciana, valora mucho los logros y resultados del aprendizaje, mientras que el grupo del Factor 2 aprecia más la experiencia del aprendizaje o el futuro uso de los conocimientos adquiridos. Dörnyei $(2005,2009)$ indica que 'el yo obligatorio de L2' se relaciona estrechamente con las obligaciones y deberes de los aprendientes, lo cual se asemeja mucho a la motivación instrumental. Sin embargo, 'el yo ideal de L2' tiene mucho que ver con los deseos de los alumnos: llegar a ser hablante de la L2, como una versión renovada del concepto de integratividad.

Llegados a este punto, podemos decir que los estudiantes del Factor 1 están más influidos por 'el yo obligatorio de L2' y prefieren dedicar más tiempo y esfuerzo al estudio del inglés, apreciando más los posibles éxitos gracias al dominio de la lengua española en vez de a otras lenguas extranjeras, ya que estas las ven como una obligación. Además, quieren dominar las lenguas extranjeras, ya sea el inglés o el español, con el fin de seguir un itinerario marcado por otras personas importantes de su entorno y por evitar cualquier tipo de fracaso en la vida. Es decir, se identifican con estos idiomas para cumplir con las obligaciones de su rol social. Al igual que en el caso de Henry (2014), el inglés en China también tiene una posición social alta y una presencia amplia, lo cual puede funcionar como una referencia normativa y convertirse en una influencia negativa para otras LLEE. En nuestro caso, el aprendizaje del inglés y la motivación presentada por los estudiantes chinos frenan los alicientes de conocer el español, debido a que ocupan demasiado tiempo y recursos en el otro idioma. Esto corresponde con el resultado de las investigaciones de Henry 
(2014) y Dörnyei $(2005,2009)$. Cuando los estudiantes se vean afectados por 'el yo obligatorio de L2', la contención producida por el inglés será muy relevante, tal y como reflejan los estudiantes del Factor 1.

Por otro lado, encontramos que, aunque a los aprendientes del Factor 1 les encantan los productos culturales ingleses, se limitan a los del Renio Unido y Estados Unidos, y no conocen la cultura de otros países angloparlantes. En cierto modo, estos dos países tienen una excesiva difusión entre los estudiantes chinos, lo que conduce a que 'el yo obligatorio de L2' solo tenga una referencia cultural que consultar, sin otro tipo de perspectivas ni pensamientos críticos. Al contrario de lo que ocurre con la invasión de los productos culturales anglo-americanos, la cultura y civilización del mundo hispánico son casi desconocidos para los estudiantes chinos. Incluso podemos decir que son algo local y no llega a tener una excesiva influencia, al contrario de lo que ocurre en el resto del mundo. Por lo tanto, la lengua española, en comparación con la inglesa, tendría un menor impacto cultural y social, provocando que los alumnos chinos no se sientan tan obligados en su motivación del 'yo obligatorio de L2', lo que coincide con el resultado de la investigación de Huang et al. (2015).

En cuanto a los estudiantes del Factor 2, aunque no se esfuerzan tanto en el aprendizaje del inglés, ya que no tienen un fuerte aliciente relacionado con los logros académicos, y siguen considerando el conocimiento de esta lengua como una tarea importante y necesaria durante su época universitaria. Esto se debe a la imposibilidad de ignorar la importante posición que ocupa el inglés en la sociedad china. Por ello, dedican más tiempo y esfuerzo al estudio del español que al inglés y muestran un gran interés por el idioma latino y su cultura, lo que representa 'el yo ideal de L2'. Lo curioso es que este grupo no cuenta con una motivación verdaderamente integrativa, es decir, 'el yo ideal de L2' tampoco les influye mucho. Los resultados del estudio revelan que aprenden español no porque crean que esta lengua sea útil para el mercado laboral, como ha señalado González Puy (2006), ni porque tengan planes profesionales o de vida muy concretos que incluyan ir a países hispanohablantes para integrarse en su sociedad. Lo aprenden porque esta lengua posee un halo misterioso y exótico que les puede representar una experiencia diferente que la del estudio del inglés. Esta razón funciona para ambos factores.

De hecho, su motivación para aprender español no es exclusiva para este idioma, sino que ocurre lo mismo con cualquier otra lengua extranjera. Así, lo que de verdad necesitan los estudiantes es una competencia multilingüe, y el español solo es un apoderado en este caso. Lo que atrae y estimula a los miembros del Factor 2 es el propio proceso de aprendizaje de este idioma, o sea, el contexto de estudio. La experiencia de aprender esta lengua, por ejemplo, con la influencia positiva de su profesora, les ayuda a conocer mejor los países hispanohablantes y la importancia de este idioma en el mundo, por lo que deciden seguir su estudio y conocer poco a poco su cultura y civilización. 
Sin duda alguna, la motivación del aprendizaje de esta lengua se relaciona estrechamente con el papel que juega el contexto, como dicen Huang et al. (2015). Por lo tanto, no podemos obviar la situación actual del español en China. Un idioma muy importante y significativo en la comunidad internacional y que no se valora tanto como debería, funcionando, incluso, como un elemento culturalmente atractivo, sin que la sociedad conozca su amplio uso y su importante identidad en el mundo global. Esto merece nuestra atención y reflexionar sobre ello con el fin de que la sociedad china tenga un conocimiento correcto y profundo sobre el español y la sociedad hispánica.

\section{CONCLUSIONES}

En el presente trabajo, mediante un experimento con metodología Q y una entrevista hecha a los participantes, hemos sacado las siguientes conclusiones:

A. Se muestra que existen dos tipos de motivaciones para el estudio simultáneo del inglés y el español en China: uno es solo ‘inglés' y el otro, ‘multilingüe'. Parte de los estudiantes chinos que aprenden lenguas extranjeras, especialmente, el inglés, se identifican por su posición social en un determinado entorno y se sienten impulsados por las expectativas sociales gracias al 'yo obligatorio de L2'. Sin embargo, hay otros alumnos que se interesan por las LLEE, tanto por el inglés como por el español, por una motivación mixta de disposición del 'yo ideal de L2' y la experiencia del aprendizaje e, incluso, por el interés cultural. Cabe destacar también que nuestro experimento revela que los dos tipos de 'auto-guías' de Dörnyei contribuyen de forma muy diferente en la adquisición del conocimiento de las lenguas extranjeras entre los aprendientes chinos.

B. La disposición motivacional y actitudinal está muy relacionada con la posición social y el conocimiento existente de la lengua en la comunidad China. A diferencia de otros estudios anteriores que proponen la posición cada día más preeminente del español en China, encontramos que la difusión de la lengua española y su cultura en esta región, incluso en Asia Oriental, sigue siendo bastante deficiente por la contención que representa el inglés. Es necesaria más información que dé más importancia al español, en especial, sobre su imprescindible papel en la política y economía mundial. De esta forma, se podrá ampliar el horizonte de los alumnos en el estudio de LLEE y se impulsará la comunicación entre Asia y los países hispanohablantes. No obstante, de nuestras conclusiones se extrae que los alumnos chinos ya empiezan a perseguir el multilingüismo, aunque aún no de forma clara.

A pesar de las limitaciones que pueda tener el presente estudio, por ejemplo, con el enfoque centrado únicamente en los estudiantes especializados en inglés, y la falta de una muestra más amplia, nos permite llegar a significativas conclusiones: primero, nos muestra la disposición motivacional y actitudinal de los estudiantes chinos hacia el aprendizaje de la lengua española; segundo, aflora el hecho de que los alumnos, pese a 
su fuerte motivación de aprender inglés, no lo toman como uno de los recursos multilingües, sino que lo utilizan como una manera de alcanzar el éxito académico y su propia autosatisfacción, y tercero, también revela la relación que hay entre la motivación de la L2 (inglés) y la L3 (español) (en el contexto asiático, el inglés es la lengua extranjera de mayor influencia y frena la motivación de los estudiantes para aprender otros idiomas). No obstante, no debemos ser muy pesimistas ante el monismo del inglés en China, dado que, según la norma de la naturaleza y el desarrollo de la sociedad, cuando algo se hace extremo, es inevitable que se mueva poco a poco al sentido contrario. Esto incluso lo podemos ver en los estudiantes 'multilingüe', que, bajo la enorme presión del inglés, empiezan a buscar otro camino que les permita tener acceso a un mundo diverso y multicultural, y a otras formas de alcanzar su autorealización, entre las cuales se encuentra el aprendizaje simultáneo de varias lenguas extranjeras.

\section{REFERENCIAS BIBLIOGRÁFICAS}

Apple, M. T., Da Silva, D. \& Fellner, T. (2013). Language learning motivation in Japan. Bristol: Multilingual Matters.

Apple, M. T., Da Silva, D. \& Fellner, T. (2016). L2 selves and motivations in Asian contexts. Bristol: Multilingual Matters.

Bolton, K. \& Graddol, D. (2012). English in China today. English Today, 28(3), 3-9.

Brown, R. Q. (1995). Q methodology as the foundation for a science of subjectivity. Ponencia presentada en la $11^{\text {th }}$ International Conference of the International Society for the Scientific Study of Subjectivity, College of Medicine, University of Illinois, USA.

Busse, V. (2017). Plurilingualism in Europe: Exploring attitudes towards English and other European languages among adolescents in Bulgaria, Germany, the Netherlands, and Spain. Modern Language Journal, 101(3), 566-582.

Cabau-Lampa, B. (2007). Mother tongue plus two European languages in Sweden: Unrealisitc educational goal? Language Policy, 6, 333-358.

Carrai, D. (2009). Motivación y abandono en el aprendizaje del español en Noruega. I: La motivación. Ponencia prensentada en el III Congreso Internacional: La enseñanza del español en tiempos de crisis, Cádiz, España.

Cenoz, J. \& Genesee, F. (1998). Psycholinguistic perspectives on multilingualism and multilingual education. En J. Cenoz \& F. Genesee (Eds.), Beyond bilingualism: Multilingual and multilingual education (pp. 16-32). Clevedon: Multilingual Matters. 
Cenoz, J. \& Jessner, U. (2000). English in Europe: The acquisition of a third language. Clevedon: Multilingual Matters.

Coffey, S. (2018). Choosing to study modern foreign languages: Discourses of value as forms of cultural capital. Applied Linguistics, 39(4), 462-480.

Coleman, J. A., Galaczi, A. \& Astruc, L. (2007). Motivation of UK school pupils towards foreign languages: A large-scale survey at Key State 3. Language Learning Journal, 35(2), 245-281.

Crystal, D. (2003). English as a global language (2nd ed.). Cambridge, Inglaterra: Cambridge University Press.

Csizér, K. \& Dörnyei, Z. (2005a). The internal structure of language learning motivation and its relationship with language choice and learning effort. Modern Language Journal, 89, 19-36.

Csizér, K. \& Dörnyei, Z. (2005b). Language learners' motivational profiles and their motivated learning behavior. Language Learning, 55(4), 613-659.

Csizér, K. \& Lukács, G. (2010). The comparative analysis of motivation, attitudes and selves: The case of English and German in Hungary. System, 38(1), 1-13.

Dörnyei, Z. (2005). The psychology of the language learner: Individual differences in second language acquisition. Mahwah, NJ: Lawrence Erlbaum.

Dörnyei, Z. (2009). The psychology of second language acquisition. Oxford: Oxford University Press.

Dörnyei, Z. \& Csizér, K. (2002). Some dynamics of language attitudes and motivation: Results of a longitudinal nationwide survey. Applied Linguistics, 23, 421-462.

Dörnyei, Z. \& Ushioda, E. (2009). Motivation, language identities and the L2 self: A theoretical overview. En Z. Dornyei \& E. Ushioda (Eds.), Motivation, language identity, and the L2 self (pp. 1-8). Bristol: Multilingual Matters.

Dörnyei, Z. \& Ushioda, E. (2011). Teaching and researching motivation (2nd ed.). Edinburgh Gate, UK: Pearson Education Limited.

Dörnyei, Z. \& Al-Hoorie, A. (2017). The motivational foundation of learning languages other than Global English: Theoretical issues and research directions. Modern Language Journal, 101(3), 455-468.

Dörnyei, Z., Csizér, K. \& Németh, N. (2006). Motivation, language attitudes and globalization: A Hungarian perspective. Clevedon: Multilingual Matters.

Espí, M. J. \& Azurmendi, M. J. (1996). Motivación, actitudes y aprendizaje del español como lengua extranjera. RESLA, 11, 63-76. 
Feng, A. W. (2009). English in China: Convergence and divergence in policy and practice. AIL A Review, 22, 85-102.

Fonseca C. \& García, L. (2010). Aprender español en USA: Los medios de comunicación como motivación social. Comunicar, 34, 145-153.

Gao, X. (2008). You had to work hard 'cause you didn't know whether you were going to wear shoes or straw sandals!'. Journal of Language, Identity and Education, 8, 169-187.

Gardner, R. C. \& Lambert, W. E. (1972). Attitudes and motivation in second-language learning. Rowley, Massachusetts: Newbury House Publishers.

Graddol, D. (2006). English next: Why global English can mean the end of "English as a foreign language”. Londres: British Council.

González Puy, I. (2006). El español en China. El español en el mundo. Anuario del Instituto Cervantes 2006-2007, 133-142.

Henry, A. (2010). Contexts of possiblity in simultaneous language learning: Using the L2 motivational self system to assess the impact of global English. Journal of Multilingual and Multicultural Development, 31(2), 149-162.

Henry, A. (2011). Examining the impact of L2 English on L3 selves: A case study. International Journal of Multilingualism, 8(3), 235-255.

Henry, A. (2014). The motivational effects of crosslinguistic awareness: Developing third language pedagogies to address the negative impact of the L2 on the L3 self-concept. Innovation in Language Teaching and Learning, 8(1), 1-19.

Henry, A. (2015). The dynamics of L3 Motivation: A longitudinal interview/observation-based study. En Z. Dörnyei, P. D. MacIntyre \& A. Henry (Eds.), Motivational Dynamics in Language Learning (pp. 315-341). Bristol: Multilingual Matters.

Herdina, P. \& Jessner, U. (2002). A dynamic model of multilingualism: Perspectives of change in psycholinguistics. Clevedon: Multilingual Matters.

Higgins, E. T. (1987). Self-discrepancy: A theory relating self and affect. Psychological Review, 94, 319-340.

Hu, G. W. (2003). English language teaching in China: Regional differences and contributing factors. Journal of Multilingual and Multicultural Development, 24(4), 290-318. 
Huang, H. T., Hsu, C. C. \& Chen, S. W. (2015). Identification with social role obligations, possible selves, and L2 motivation in foreign language learning. System, 51, 28-38.

Instituto Cervantes. (2016). El español: Una lengua vivia. Informe 2016 [en línea]. Disponible en: http://www.cervantes.es/imagenes/File/prensa/EspanolLenguaViva16.pdf

Irie, K. \& Ryan, S. (2015). Study abroad and the dynamics of change in learner L2 selfconcept. En Z. Dörnyei, P. D. MacIntyre \& A. Henry (Eds.), Motivational dynamics in language learning (pp. 343-367). Bristol: Multilingual Matters.

Jessner, U. (2006). Linguistic awareness in multilinguals: English as a third language. Edinburgh, UK: Edinburgh University Press.

Jessner, U. (2008). A DST model of multilingualism and the role of metalinguistic awareness. Modern Language Journal, 92(2), 270-283.

Lam, A. S. L. (2002). English in education in China: Policy changes and learners' experiences. World Englishes, 21(2), 245-256.

Lamb, M. (2004). Integrative motivation in a globalizing world. System, 32, 3-19.

Lamb, M. (2009). Situating the L2 self: Two Indonesian school learners of English. En Z. Dörnyei \& E. Ushioda (Eds.), Motivation, language identity and the L2 Self (pp. 229-247). Bristol: Multilingual Matters.

MacIntyre, P. D., Mackinnon, S. P. \& Clément, R. (2009). The baby, the bathwater, and the future of language learning motivation research. En Z. Dörnyei \& E. Ushioda (Eds.), Motivation, language identity and the L2 Self (pp. 43-65). Bristol: Multilingual Matters.

MacIntyre, P. D., MacKay, E., Ross, J. \& Abel, E. (2017). The emerging need for methods appropriate to study dynamic systems: Individual differences in motivational dynamics. En L. Ortega \& Z. Han (Eds.), Comlexity theory and language development: In celebration of Diane Larsen-Freeman (pp. 97-122). Amsterdam: John Benjamins.

Markus, H., \& Nurius, P. (1986). Possible selves. American Psychologist, 41, 954-969.

Mercer, S. (2011a). Language learner self-concept: Complexity, continuity and change. System, 39(3), 335-346.

Mercer, S. (2011b). Understanding learner agency as a complex dynamic system. System, 39(4), 427-436. 
Minera, L. E. (2009). El papel de la motivación y las actitudes en el aprendizaje de ELE en un contexto de enseñanza formal para adultos alemanes. Revista Nebrija de Lingüistica Aplicada a la Enseñanza de las Lenguas, 6, 58-73

Ortega, L. \& Byrnes, H. (2008). The longitudinal study of advanced L2 capacities. Nueva York: Routledge.

Pan, L. (2011). English language ideologies in the Chinese foreign language education policies: A world-system perspective. Language Policy, 10, 245-263.

Pan, L. \& Block, D. (2011). English as a "global language" in China: An investigation into learners' and teachers' language beliefs. System, 39, 391-402.

Prou, O., Le Nedic, T. \& Amslem T. (2009). La méthodologie Q et l'étude de la subjectivité. (Curso de Methodologies quantitatives, Prof. Benavent, Master 2GDO, Ecole des Mines, Paris, mimeo, 14) [en línea]. Disponible en: http://christophe.benavent.free.fr/IMG/pdf/GDO_2009_Methode_Q_et_a pplication.pdf

Segalowitz, N. \& Freed, B. F. (2004). Context, contact and cognition in oral fluency acquisition: Learning Spanish in at home and study abroad contexts. Studies in Second Language Acquisition, 26, 173-199.

Siridetkoon, P. \& Dewaele, J.-M. (2017). Ideal self and ought-to self of simultaneous learners of multiple foreign languages. International Journal of Multilingualism, 15(4), 313-328. Doi: 10.1080/14790718.2017.1293063

Taguchi, T., Magid, M. \& Papi, M. (2009). The L2 motivational self system among Japanese, Chinese, and Iranian Learners of English: A Comparative Study. En Z. Dörnyei \& E. Ushioda (Eds.), Motivation, Language Identity and the L2 Self (pp. 66-97). Bristol: Multilingual Matters.

Valencia Vallejo, N. G. (2003). La metodología Q: Más que una técnica de investigación. Tecné, episteme y didaxis: Revista de la Facultad de Ciencia y Tecnología, $13,144-153$

Watts, D. S. (2015). Develop a Q methodological study. Education for Primary Care, 26(6), 435-437.

Watts, S. \& Stenner, P. (2012). Doing $Q$ methodological reserch: Theory, method and interpretation. Londres: Sage.

Wei, R. \& Su, J. (2012). The statistics of English in China. English Today, 28(3), 10-14.

Yang, Y. \& Montgomery, D. (2013). Gaps or bridges in multicultural teacher education: A Q study of attitudes toward student diversity. Teaching and Teacher Education, 30, 27-37. 
Yashima, T. (2002). Willingness to communicate in a second language: The Japanese EFL context. Modern Language Journal, 86, 54-66.

Yashima, T. (2009). International posture and the ideal L2 self in the Japanese EFL context. En Z. Dörnyei \& E. Ushioda (Eds.), Motivation, language identity and the L2 Self (pp. 144-163). Bristol: Multilingual Matters.

Zheng, S. (2015). La investigación de la enseñanza de español en universidades chinas. Beijing: Foreign Language Teaching and Research Press.

\section{ANEXO I}

Lista de los 60 enunciados

\begin{tabular}{|c|c|}
\hline & Traducciones al español de los enunciados \\
\hline 1 & Prefiero dedicar más tiempo y esfuerzo al estudio del inglés. \\
\hline 2 & Prefiero dedicar más tiempo y esfuerzo al estudio del español. \\
\hline 3 & Dedico la mayor parte de mi tiempo libre al estudio del inglés. \\
\hline 4 & Dedico la mayor parte de mi tiempo libre al estudio del español. \\
\hline 5 & Continuaré aprendiendo inglés en el futuro. \\
\hline 6 & Continuaré aprendiendo español en el futuro. \\
\hline 7 & $\begin{array}{l}\text { Siempre aseguro suficiente tiempo para el estudio de inglés, y el resto, lo } \\
\text { dedico al español. }\end{array}$ \\
\hline 8 & $\begin{array}{l}\text { Siempre asigno una gran parte de mi tiempo, primero al estudio del español, y } \\
\text { el resto, al inglés. }\end{array}$ \\
\hline 9 & $\begin{array}{l}\text { Me parece que hablo bastante bien inglés, así que tengo tiempo y esfuerzo } \\
\text { para aprender otra lengua extranjera-español. }\end{array}$ \\
\hline 10 & $\begin{array}{l}\text { Puedo imaginar que en el futuro podré usar el inglés sin ningún problema en } \\
\text { algún trabajo o en la vida cotidiana. }\end{array}$ \\
\hline 11 & $\begin{array}{l}\text { Puedo imaginar que en el futuro podré usar el español sin ningún problema } \\
\text { en algún trabajo y en la vida cotidiana. }\end{array}$ \\
\hline 12 & Puedo imaginar que podré usar el inglés como los angloamericanos. \\
\hline 13 & Puedo imaginar que podré usar el español como los españoles. \\
\hline 14 & $\begin{array}{l}\text { Si algún día voy a los países hispanohablantes, prefiero hablar en inglés con } \\
\text { los nativos si ellos pueden hablar inglés. }\end{array}$ \\
\hline 15 & $\begin{array}{l}\text { Si algún día voy a los países hispanohablantes, prefiero hablar en español con } \\
\text { los nativos a pesar de que sepan hablar inglés. }\end{array}$ \\
\hline 16 & Tengo que aprender bien el inglés, si no, afectará a mis calificaciones. \\
\hline 17 & $\begin{array}{l}\text { Tengo que aprender bien inglés, si no, no podré encontrar un buen trabajo en } \\
\text { el futuro }\end{array}$ \\
\hline 18 & Tengo que aprender bien español, si no, afectará a mis calificaciones. \\
\hline 19 & $\begin{array}{l}\text { Tengo que aprender bien español, si no, no podré encontrar un buen trabajo } \\
\text { en el futuro. }\end{array}$ \\
\hline 20 & Como hablo bien inglés, no es importante si no puedo aprender bien español. \\
\hline 21 & $\begin{array}{l}\text { Aunque hablo bastante bien el inglés, tengo que aprender bien el español. Si } \\
\text { no, los otros pueden pensar que no soy capaz de aprender LLEE. }\end{array}$ \\
\hline
\end{tabular}




\begin{tabular}{|c|c|}
\hline 22 & $\begin{array}{l}\text { Aunque hablo bastante bien inglés, tengo que aprender bien el español, } \\
\text { porque me facilitará la búsqueda de un buen trabajo. }\end{array}$ \\
\hline 23 & Me gustan mucho las clases de inglés que estoy cursando. \\
\hline 24 & Me gustan mucho las clases de español que estoy cursando. \\
\hline 25 & Los materiales didácticos de inglés me parecen interesantes. \\
\hline 26 & Los materiales didácticos de español me parecen interesantes. \\
\hline 27 & Ya llevo bastante tiempo aprendiendo inglés, y me parece un poco aburrido. \\
\hline 28 & Me parece que aprender español es muy novedoso. \\
\hline 29 & Siempre me parece que el inglés es más fácil cuando estudio español. \\
\hline 30 & Me parece que aprender español es útil para el estudio del inglés. \\
\hline 31 & Siempre hago comparaciones entre español e inglés. \\
\hline 32 & $\begin{array}{l}\text { Al encontrarme con dificultades en el estudio del español, siempre pienso: } \\
\text { "No pasa nada; por lo menos, hablo bien el inglés." }\end{array}$ \\
\hline 33 & $\begin{array}{l}\text { Al encontrarme con dificultades en el estudio del inglés, siempre pienso: "No } \\
\text { pasa nada, no sería importante si no pudiera aprender bien las lenguas } \\
\text { extranjeras." }\end{array}$ \\
\hline 34 & Hoy en día, una persona debe aprender más de una lengua extranjera. \\
\hline 35 & Siempre envidio a las personas que pueden hablar varias lenguas. \\
\hline 36 & $\begin{array}{l}\text { Saber hablar por lo menos una lengua extranjera representa que él o ella } \\
\text { reciba una buena educación. }\end{array}$ \\
\hline 37 & En este mundo se necesita muchas diferentes lenguas, no solo el inglés. \\
\hline 38 & $\begin{array}{l}\text { Como el inglés es la lengua franca o vehicular en todo el mundo, sería } \\
\text { suficiente aprender solo esta lengua. }\end{array}$ \\
\hline 39 & $\begin{array}{l}\text { Me parece que todo el mundo puede hablar inglés ahora, por eso aprender el } \\
\text { español puede ser una ventaja. }\end{array}$ \\
\hline 40 & $\begin{array}{l}\text { Necesitamos aprender inglés para participar en los asuntos de la comunidad } \\
\text { internacional. }\end{array}$ \\
\hline 41 & $\begin{array}{l}\text { Me parece que aprender inglés es aprender el inglés estándar de Inglaterra o } \\
\text { de EEUU. }\end{array}$ \\
\hline 42 & $\begin{array}{l}\text { Estudio español en vez de francés, alemán o japonés, porque el español tiene } \\
\text { una posición importante en la comunidad internacional. }\end{array}$ \\
\hline 43 & El español me parece muy interesante. \\
\hline 44 & He decidido aprender español porque es una lengua relativamente fácil. \\
\hline 45 & Puedo conocer todo tipo de consultas si domino el inglés. \\
\hline 46 & $\begin{array}{l}\text { Aprendo inglés porque tengo ganas de comunicarme con las personas que no } \\
\text { hablen chino. }\end{array}$ \\
\hline 47 & $\begin{array}{l}\text { Aprendo español para conocer mejor la situación de los países } \\
\text { hispanohablantes. }\end{array}$ \\
\hline 48 & $\begin{array}{l}\text { Me gustan los productos culturales de los países anglo-americanos, tales como } \\
\text { la música, las series o las películas. }\end{array}$ \\
\hline 49 & Me gusta mucho el estilo de vida de los angloamericanos. \\
\hline 50 & $\begin{array}{l}\text { Me gusta la cultura de los países hispanohablantes, incluidos el fútbol, la } \\
\text { danza, la música, etc. }\end{array}$ \\
\hline 51 & Me interesa mucho la cultura de los hispanohablantes en EEUU. \\
\hline 52 & Espero que algún día pueda ser personas a estilo de los angloamericanos. \\
\hline 53 & Espero que algún día pueda ser personas a estilo de los hispanohablantes. \\
\hline 54 & Creo que para adquirir una lengua también hace falta conocer su cultura. \\
\hline
\end{tabular}




\begin{tabular}{|l|l|}
\hline 55 & $\begin{array}{l}\text { Espero ir algún día a los países anglo-americanos para hacer intercambios o } \\
\text { para vivir. }\end{array}$ \\
\hline 56 & $\begin{array}{l}\text { Espero que algún día pueda ir a los países hispanohablantes para hacer } \\
\text { intercambios o para vivir. }\end{array}$ \\
\hline 57 & $\begin{array}{l}\text { Me parece que la posición internacional de los países latinoamericanos es cada } \\
\text { día más alta. }\end{array}$ \\
\hline 58 & $\begin{array}{l}\text { Me parece que la posición de los hispanohablantes en EEUU es cada día más } \\
\text { alta. }\end{array}$ \\
\hline 59 & $\begin{array}{l}\text { En comparación con los extranjeros de otros países anglohablantes, me } \\
\text { gustan más los angloamericanos. }\end{array}$ \\
\hline 60 & $\begin{array}{l}\text { Me gustaría poder comunicarme con los españoles o latinoamericanos en } \\
\text { español. }\end{array}$ \\
\hline
\end{tabular}

\section{ANEXO II}

Lista de las preguntas en la entrevista (traducciones al español)

1. ¿Qué opiniones tiene sobre el inglés y el español?

-- ¿Qué opiniones tiene sobre las dos culturas? ¿Le van a afectar su estudio de las dos lenguas?

-- ¿Cómo conoce la posición de las dos lenguas en la comunidad internacional y en nuestro país?

-- ¿Tiene algún sentido específico para usted el español y su cultura? ¿Y el inglés?

2. ¿Tiene plan de asistir a algún examen oficial de español o inglés para conseguir un certificado? Si lo tiene, ¿cómo es su plan?

-- ¿Por qué quiere conseguir un certificado de lengua?

$--¿ V a$ a utilizar el certificado para buscar trabajo?

-- ¿Cree que va a encontrar algún trabajo relacionado con el español?

3. En cuanto al intercambio o estudio en el futuro, ¿tiene algún plan?

-- ¿Quiere hacer el intercambio en los países hispanohablantes o en los angloamericanos? ¿Por qué?

-- ¿Tiene el plan de ir a estudiar un máster o doctorado en el futuro? ¿Qué país prefiere? ¿Va a considerar los países hispanohablantes?

-- ¿Le gustaría hacerse amigo de algunos extranjeros?

4. ¿Qué opiniones tiene sobre el estudio de varias lenguas extranjeras?

$--¿$ Cree que en el mundo actual hacen falta diferentes lenguas?

-- ¿Por qué elige el español como su L3? ¿Ha considerado otras lenguas extranjeras?

-- ¿La capacidad de hablar varias LLEE le va a ayudar en el futuro? ¿Cómo le ayuda?

\section{NOTAS}

${ }^{1}$ Z-Score en inglés. Se calcula automáticamente por el software PQMethod. 